\title{
Comparative study of floristic diversity along altitude in the northern slope of the central Alborz Mountains, Iran
}

\author{
HALIME MORADI", FARIDEH ATTAR ${ }^{\nu \nu}$ \\ Central Herbarium of Tehran University, Department of Botany, School of Biology, College of Science, University of Tehran. 16 ${ }^{\text {th }}$ Azar St., Enghelab \\ Sq., Tehran 1417466191, Iran. Tel.: +98-21-61112968, ‘email: hlh.moradi@gmail.com; ” fattar@khayam.ut.ac.ir
}

Manuscript received: 6 November 2018. Revision accepted: 29 December 2018.

\begin{abstract}
Moradi H, Attar F. 2019. Comparative study of floristic diversity along altitude in the northern slope of the central Alborz Mountains, Iran. Biodiversitas 20: 305-312. The Alborz is one of two main mountain chains in the north of Iran. The flora and vegetation of the sub-alpine and alpine zone of the central Alborz are less known comparing to the vegetation of lower altitudes with Hyrcanain forests. In this study, floristic composition and life-form spectra were investigated along an altitudinal transect ranging from $2000 \mathrm{~m}$ to the alpine and sub-nival peak of Mt. Rostam-Nisht at $4500 \mathrm{~m}$. We compared the floristic diversity of the studied transect with the data obtained from an earlier studied transect in Kheyrud forest. A total of 299 taxa were found in the survey which showed high species diversity in the area. In addition, our results showed remarkable difference in life form categories between lower altitudes (Hyrcanian forests) and sub-alpine and alpine zones.
\end{abstract}

Keywords: Alpine flora, biodiversity, Caspian forest, conservation, elevation

\section{INTRODUCTION}

Biodiversity includes the variety of species, genes they contain, the communities and ecosystems of which they are a part (McNeely et al. 1990). In a particular region, it indicates the richness in floristic composition, i.e., the diversity of species which in any given plant community is often termed as species richness (Van der Maarel 2005). The rapid loss in species and habitats, and changing the pattern of vegetation due to various biotic and abiotic factors have imposed the assessment of diversity. So, the need for floristic knowledge as a base for biodiversity conservation is required. This knowledge can help to monitor the species loss, distribution of species and the effect of anthropogenic pressures on species habitat destruction (Pant and Samant 2012). Such studies can finally locate hot spots and areas with high biodiversity for conservation (Phillips et al. 2003).

Floristic studies are undertaken by many researchers following various aspects of species diversity. E.g. studying the diversity of plant life forms in different vegetation types or communities (Siadati et al. 2010; Naqinezhad et al. 2015) afford direct structural components of vegetation stands and explaining vegetation structure (Box 1981). In this regard, assessment of biodiversity along altitudes can reduce plant community complexity. This is because of steep environmental gradients at relatively short distances (Körner 2007) which simply disclose the latitudinal changes of diversity (Mc Cain 2007). Some aspects of mountains such as spatial scale, power of theoretical tests and variability of taxonomic signal make them ideally suited for examining biodiversity (Körner
2000; Mc Cain 2009; Qiong et al. 2012) and attract many researchers to compare them floristically.

The Alborz, the best-known mountain range in Iran has been poorly investigated, particularly ecologically and botanically in alpine areas (Noroozi et al. 2008). In Iran, the studies related to vegetation structure as well as the flora mainly focused on forests (e.g. Jafari and Akhani 2008; Siadati et al. 2010; Jafari et al. 2013; Naqinezhad et al. 2015; Gholizadeh et al. 2017), while few studies were conducted in the regions above $2000 \mathrm{~m}$ (e.g. Akhani et al. 2013; Mahdavi et al. 2013; Noroozi et al. 2011; Moradi et al. 2017).

The current investigation is a part of a project to study the vegetation and ecological properties of sub-alpine and alpine areas along an altitudinal transect in high mountains of the central Alborz. The main objective of this study was to provide a floristic list of the vascular plants in the area. This can lead to complement the information about the locality of the species, specifically those with restricted altitudinal distribution. Besides, it provides more knowledge on biodiversity conservation. Moreover, we compared the floristic composition of our studied transect with a transect located in Kheyrud forest $(50-2200 \mathrm{~m})$ where is belonged to the Hyrcanian forests. The Hyrcanian forest is a unique natural closed-canopy deciduous forest with a gradient of floristic changes (Moradi et al. 2016) in northern slope of the Alborz from Caspian Sea level to the altitudes at $<2800 \mathrm{~m}$. The findings should provide insights to (i) the potential of biodiversity within the central Alborz Mountains, and (ii) a comparison between the flora and life forms of our studied area and Kheyrud forest. 


\section{MATERIALS AND METHODS}

\section{Study area}

The Alborz extends at the southern shore of the Caspian Sea from the Ararat mountainous range, in the border of Armenia in the west to Hindukush range in Afghanistan to the east. It acts as a natural barrier between the Caspian Sea and the Central Plateau of Iran. The Alborz is topographically divided into eastern, central and western Alborz with maximal altitudes between $3000-3500 \mathrm{~m}$ and the valleys with a minimum of around $2000 \mathrm{~m}$. It includes several peaks in the central Alborz with more than $4000 \mathrm{~m}$, e.g., Mt. Damavand (5671 m) and Mt. Alamkuh (4840 m) (Khalili1973).

The Alborz has two distinct climatic regimes for northern and southern slopes. The climatic information delineates a dry to semi-dry climate with a semi Mediterranean rainfall regime mainly in winter on the southern slope. Conversely, in the northern slope, there is a humid and sub-humid climate with maximum precipitation in autumn and winter and a relative minimum rainfall in spring with no real dry season. The climate is cold and subhumid in lower altitudes $(2100 \mathrm{~m})$ and cold and dry in the sub-alpine and alpine zones (2400-4000 m) (Khalili 1973).

The northern and southern slopes divide the Alborz floristically. The southern slope is covered by dry IranoTuranian steppic plant assemblages, while the Hyrcanian forest encompasses the northern slope in lower altitudes $(<2800 \mathrm{~m})$ and is characterized by temperate broad-leaved deciduous trees (Zohary 1973; Frey and Probst 1986). Toward higher altitudes, in sub-alpine areas, the grasslands and steppic vegetation replace the Hyrcanian forest, which is mainly divided into tall herbaceous vegetation (Noroozi 2014), grassland with scattered trees and grasslands with thorn cushion (Frey and Probst 1986). Thorny cushions are mostly found at sub-alpine altitudes with lowest temperature and long snow cover duration (Klein 1987) followed by chasmophytic and mobile screen species at alpine and nival areas in rocky habitats and screes (Klein 1982; Noroozi et al. 2008; Noroozi 2014).

Our studied transect is located on the northern slope of the central Alborz in the Mazandaran province between $\mathrm{N}$ $36^{\circ} 26^{\prime} 16.1^{\prime \prime}$, E $051^{\circ} 03^{\prime} 23.2^{\prime \prime}$ and N $36^{\circ} 24^{\prime} 05.9^{\prime \prime}$, E $050^{\circ}$ $57^{\prime} 43.7^{\prime \prime}$. It consists of starts from $2000 \mathrm{~m}$ a.s.l. just above the timberline until $4500 \mathrm{~m}$ to the peak of Mt. RostamNisht (Figure 1).

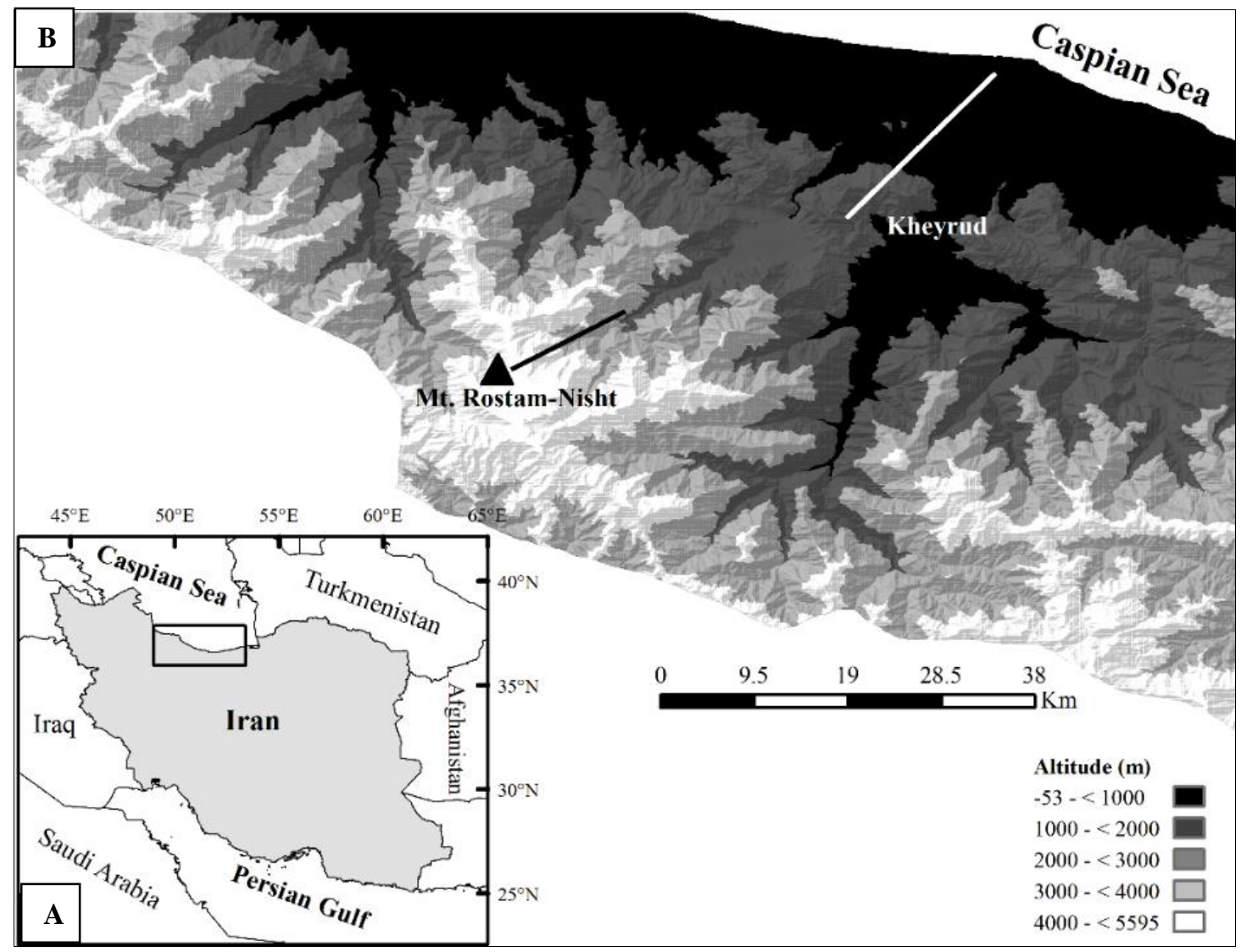

Figure 1. The study area. A. The location of Iran and the Alborz Mountain in the south of Caspian Sea. B. The western part of the northern slope of the central Alborz; Black line shows the studied altitudinal transect along Rostam-Nisht peak, and the white line shows the altitudinal transect along Kheyrud forest 


\section{Vegetation sampling}

Field sampling was performed during the growing season (summer and spring) of 2013 to 2015 in a total of 76 vegetation plots. Three $10 \times 10 \mathrm{~m}$ vegetation plots were allocated in each $100 \mathrm{~m}$ elevational interval along an altitudinal transect ranging from $2000 \mathrm{~m}$ up to $4500 \mathrm{~m}$, in sub-alpine, alpine and nival areas. We took only one vegetation plot at $4500 \mathrm{~m}$ because of little space at the peak. The data related to the location, elevation, slope degree and exposition of the plots were noted. The voucher specimens were deposited in the central Herbarium of Teheran University (TUH) and were identified based on Flora Iranica (Rechinger 1963-2010), Flora of Iran (Assadi et al. 1988-2015) and Flora of Iraq (Townsend et al. 19661985). We followed Raunkiaer's classification (Raunkiaer 1934) to separate the life forms of the species found in our survey.

In order to compare the floristic composition of the Hyrcanian forest and steppic grasslands of the central Alborz, we considered the sub-alpine,alpine and nival areas of Mt. Rostam-Nisht and the data from an earlier studied transect in Kheyrud forest (Siadati et al. 2010). This transect is located $7 \mathrm{~km}$ east of Nowshahr in Mazandaran province, between $51^{\circ} 33^{\prime \prime} 12^{\prime}-51^{\circ} 39^{\prime \prime} 56^{\prime} \mathrm{E}$ and $36^{\circ} 33^{\prime \prime} 08^{\prime}-$ $36^{\circ} 45^{\prime \prime} 05^{\prime} \mathrm{N}$, ranging from 50 to $2100 \mathrm{~m}$ a.s.l. (Figure 1).The methods for collecting vegetation data in Kheyrud transect was similar to the transect in Mt. Rostam-Nisht including the altitudinal intervals between the plots (100 $\mathrm{m})$, the number of the plots per interval (3 plots), nomenclature and the extracted species attributes. However, the size of the plots differed between two transects $(10 \times 10 \mathrm{~m}$ in Mt. Rostam-Nisht and $20 \times 20 \mathrm{~m}$ in Kheyrud forest) because of the difference in their vegetation types (closed forests vs. grasslands).

\section{RESULTS AND DISCUSSION}

\section{Floristic diversity and the comparison of their vegetation types}

The locality of the Alborz between Hindu KushHimalaya Mountains in the east, and Anatolia and Caucasus Mountains in the west makes it so important and strategic in terms of historical, evolutionary, phytogeographical and biogeographical aspects (Noroozi et al. 2008; Naqinezhad et al. 2017). The Caucasus and Central Anatolian Mountains have been identified as the areas with a strong biogeographic connection with the Alborz (Noroozi 2008). The occurrence of a high number of vascular plant species in these mountainous ecosystems demonstrates their high floristic diversity and biodiversity importance. A total of 299 plant taxa of vascular plants were recorded along our altitudinal transect in Mt. RostamNisht (Table 1), as a representative of the sub-alpine, alpine and nival areas of the central Alborz. While, based on studies on Caucasus and literature survey, 226 species, 96 genera and 35 families were recorded in the sub-nival belt of the Caucasus within a range of 2800 (2900) - $4000 \mathrm{~m}$ (Shetekari et al. 2012). In the western and central Taurus, 180 vascular plant species were exclusive to the highest life zone which 150 species were found in altitudes higher than $3000 \mathrm{~m}$ (Parolly 2015). The study on the Naran Valley located in the western Himalayan province of the IranoTuranian region demonstrated 198 species and 68 families (Khan et al. 2012).

Although the Alborz mountain is rich in plant species, this is not applied to all altitudinal ranges, e.g., the highest number of species (58 species) was occurred in lower altitudinal ranges at $2200 \mathrm{~m}$, while, a few numbers of species (4 species) were found at $4400 \mathrm{~m}$.

The recorded species was belonged to 48 families and 168 genera, among them were four families of pteridophytes, one family of gymnosperms and 43 families of angiosperms. Eudicots with 36 families, 139 genera and 245 species were the richest group, while monocots had 7 families, 23 genera and 47 species in the studied flora (Table 2). The families Asteraceae (34 taxa, 11.37\%), Poaceae (31 taxa, 10.37), Lamiaceae (25 taxa, 8.36\%), Fabaceae (25 taxa, 8.36\%) and Caryophyllaceae (20 taxa, $9.69 \%$ ) showed the highest species richness. The families Brassicaceae (18 taxa, 6.02\%), Rosaceae (16 taxa, 5.35\%), Scrophulariaceae (15 taxa, 5.02\%), Boraginaceae (11 taxa, $3.68 \%$ ), Apiaceae $(11,3.68 \%)$ and Rubiaceae (10 taxa, $3.34 \%$ ) were the next species-rich families.

The families Asteraceae $(17,10.12 \%)$, Lamiaceae (15, $8.93 \%)$, Brassicaceae $(14,8.33 \%)$, Poaceae $(13,7.74 \%)$, Apiaceae $(11,6.55 \%)$ and Caryophyllaceae (10, 5.95\%) were the most genera rich. The genera with the highest species richness were Astragalus (13 taxa, 7.74\%), Potentilla (9 taxa, 5.36\%), Veronica (8 taxa, 4.76\%), Bromus (7 taxa, 4.17\%), Poa (7 taxa, 4.17\%) and Galium (6 taxa, 3.57\%). The genera such as Astragalus, Nepeta, Cousinia, Potentilla, Silene and Oxytropis were found to be the richest genera in the area which was reported by other researchers (e.g., Noroozi et al. 2008; Rechinger 19632010). These genera have been found in Mt. Rostam-Nisht, particularly in high altitudes. Accordingly, these genera contained a high number of endemic species, e.g., 7 out of 13 taxa of Astragalus are the endemic taxa which occurred mainly at altitudes higher than $3500 \mathrm{~m}$ in the studied area.

The variation in different groups of taxa is another reflection of high floristic diversity along lowlandmountain transects in the central Alborz. Here, a clear example is the higher occurrence of pteridophytes in Kheyrud forest (in altitudes 50-2200 m) than sub-alpine and alpine areas (2000-4500 m). The high soil humidity in wet seasons and dense canopy tree covers in forests makes the pteridophytes show a remarkable diversity (Siadati et al. 2010) in the forest, though they are few in the subalpine and alpine areas. This can simply mirror the variation of plant groups along altitude which may also reveal the climatic variation in the Alborz (Moradi et al. 2017). 
Table 1. Checklist of identified plant species at Mt. Rostam-Nisht with life form categories and the altitudinal distribution range per species

\begin{tabular}{l}
\hline Name of taxa \\
\hline Pteridophytes \\
Aspleniaceae \\
Asplenium ceterach $\mathrm{L}$. \\
Asplenium septentrionale (L.) Hoffm. \\
Dryopteridaceae \\
Dryopteris dilatata (Hoffm.) A. Gray. \\
Equisetaceae \\
Equisetum ramosissimum Desf. \\
Polypodiaceae \\
Polypodium interjectum Shivas. \\
Pteridaceae \\
Adiantum capillus-veneris L.
\end{tabular}

$\begin{array}{lc}\text { Life } & \text { Altitudinal } \\ \text { form } & \text { range }\end{array}$

$\begin{array}{lc}\text { Hem } & 2200-2800 \\ \text { Hem } & 2100\end{array}$

Hem 2800-3100

Hem 2100-2200

Hem 2100

Hem 2800

\section{Gymnosperms}

Cupressaceae

Juniperus communis L.

$2500-2600$

\section{Monocots}

Alliaceae

Allium ampeloprasum L. subsp. iranicum Ge Wendelbo.

Allium capitellatum Boiss.

Allium derderianum Regel.

Allium umblicatum Boiss.

Araceae

Arum maculatum L.

Cyperaceae

Carex divulsa Stokes.

Carex songorica Kar. \& Kir.

Iridaceae

Iris imbricata Lindl.

Iris reticulata $\mathrm{M}$. Bieb.

Ixioliriaceae

Ixiolirion tatarica Pall.

Liliaceae

Fritillaria kotschyana Herb. subsp.

kotschyana

Gagea gageoides (Zucc.) Vved.

Muscari neglectum Guss. ex Ten.

Ornithogalum bungei Boiss.

Ornithogalum orthophyllum Ten.

Puschkinia scillioides Adams.

Poaceae

Agropyron leptorum (Nevski) Grossh.

Agropyron longe aristata

Agropyron pectiniforme Roem. \& Schult.

Alopecurus textilis Boiss.

Bromus biebersteinii Roem. \& Schult.

Bromus briziformis Fisch. \& C.A.Mey.

Bromus erectus Huds.

Bromus tectorum L.

Bromus tomentosus Trin.

Bromus tometollus Boiss.

Bromus variegatus M. Bieb.

Dactylis glomerata L.

Festuca ovina $\mathrm{L}$.

Hordeum glaucum Steud.

Lolium multiflorum Lam.

Lolium perenne $\mathrm{L}$.

Lolium rigidum Gaudin.

Melica jacquemontii Decne. f. subsp.

hohenackeri (Boiss.) W.Hempel

Melica persica Kunth.

Milium vernale $\mathrm{M}$. Bieb.

Oryzopsis gracilis (Mez) Pilg.

$2000-3500$

$\mathrm{Ge} \quad 2200-3800$

Ge $2100-2900$

Hem 2200

Hem 2000-2300

Ge $\quad 2500$

Ge $\quad 2200$

Ge $\quad 2200-2600$

Hem 3100-3800
Phleum iranicum Bornm. \& Gauba

Phleum paniculatum Huds.

Poa araratica Trautv.

Poa bulbosa L.

Poa cf. апnиa L.

Poa longifolia Trin.

Poa nemoralis $\mathrm{L}$.

Poa sp.1

Poa sp.2

Trisetum flavescens (L.) P.Beauv.

Hem 2100-3000

Thr 2000-2600

Hem 2400-3400

Hem 2000-4100

Thr $\quad 4300$

Hem 2000-2700

Hem 2700

Hem 2100-4000

Hem 4400

Hem 2200-3700

\section{Eudicots}

Aceraceae

Acer campestre $\mathrm{L}$.

$\mathrm{Ph}$

2000-2200

Apiaceae

Anthriscus nemorosus (M.Bieb.) Spreng. Hem 2400-3700

Bunium cylindricum (Boiss. Hohen.) Hem

Drude in Engler \& Prantl.

Bupleurum exeltatum M.B. Hem

Cervaria cervariifolia (C.A. Mey) M. Pimen. Hem

Ferula szowitsiana D.C. var.

2100-2900

kandavanensis

Heracleum pastinacifolium C. Koch. $\quad$ Ch $\quad 2400-2900$

Laser trilobum (L.) Borkh. $\quad$ Hem

Pimpinella tragium Vill. subsp. lithophila $\mathrm{Hem}$

Prangos ulopterae DC.

Torilis radiata Moench.

$\mathrm{Ch}$

Trachydium depressum (Boiss.) Boiss. Hem

subsp. depressum

Asteraceae

Achillea aucheri Boiss. subsp. aucheri Hem 3600-3700

Achillea millefolium L. subsp. elbursensis Hem

Anthemis triumfettii (L.) DC. subsp. Hem

khorassanica

Artemisia absinthium L.

Artemisia chamaemelifolia Vill.

Artemisia melanolepis Boiss.

Ch 2000-3000

Centaurea zuvandica (Sosn.) Sosn.

Cirsium lappaceum M. Bieb. subsp. ferox

Ch 2100-3400

Hem 3700-4300

Boiss.

Cirsium vulgare (Savi.) Ten.

Ch 2100-3600

Cousinia pinarocephala Boiss.

Ch

Hem

Cousinia pterocaulos (C.A.Mey) Rech.f. Hem

Crepis multicaulis Ledeb. subsp. Hem

multicaulis

Crepis sancta (L.) Babc. subsp.

Hem

wemausensis

Crepis willemetioides Boiss. Hem

Erigeron caucasicus Stev. subsp. venustus Hem

(Botsch.) Grierson.

Helichrysum graveolens Sweet. Hem

Helichrysum plicatum DC. Hem

Helichrysum psychrophilum Boiss. Hem

Hieracium procerigenum Litw. \& Zahn. Hem

Lactuca wilmhelsiana Fisch. \& C.A.Mey.) Ch

in DC.

Leontodon hispidus L. var. Hem

mazanderanicus Rech.f.

Psygrogeton amorphoglassus (Boiss.) Hem 3900

Novopokr.

Scorzonera cinerea Boiss. Hem 2400-2500

Scorzonera radicosa Boiss.

Hem

Senecio vulcanicus Boiss. Hem

Tanacetum coccineum (Willd.) Grierson. Hem

subsp. coccineum

Tanacetum parthenium (L.) Sch. Bip. Hem

Tanacetum polycephalum Sch.Bip. subsp. Ch

duderanum

Taraxacum sp.1

Taraxacum sp.2

Taraxacum sp. 3

Hem

Hem

Hem

2000-2300

2400-3800

2000-4200

3900-4000

2000-2400

2000-2900

3900-4200

2900-3600

2500-3300

3400-4100

2300-2600

2000-2700

2500-3700

4000

3800-4200

2500-2600

2300-3000

2000-3700

2100-3700

2000-2900 


\begin{tabular}{l}
\hline Taraxacum sp.4 \\
Tragopogon gongylorrhizus Rech.f. \\
Tragopogon kotschyi Boiss. \\
Berberidaceae \\
Berberis integerrima L. \\
Bongardia chrysogonum (L.) Griseb. \\
Boraginaceae \\
Alkana frigida Boiss. \\
Cynoglossum creticum Miller. \\
Echium amoenum Fisch. \& C.A. Mey. \\
Lappula microcarpa Gürke. \\
Myosotis lithospermifolia Hornem. \\
Myosotis olympica Boiss. \\
Nonnea persica Boiss. \\
Onosma dichroanthum Boiss. \\
Rochelia disperma (L.F.) Koch. \\
Rochelia peduncularis Boiss. \\
Solenanthus stamineus J.F.Macbr.
\end{tabular}

\section{Brassicaceae}

Aethionema grandiflorum Boiss. \& Hohen. Alliaria petiolata (M. B.) Cavara \& Grande Alyssopsis mollis (Jacq.) O. E. Schulz Alyssum desertorum Stapf. var. desertorum Alyssum minus (L.) Rothm. var. micranthum (C.A. Mey.) Dudley.

Alyssum repens Baumg. var.

trichostachyum (Rupr.) Hayek.

Anchonium elichrysifolium Boiss.

Arabis caucasica Willd. subsp. caucasica Arabis sp.

Cardaria draba (L.) Desv.

Descurainia sophia (L.) Webb. in Berth.

Didymophysa aucheri Boiss.

Draba nemorosa L.

Erysimum caespitosum DC.

Erysimum cuspidatum D.C

Isatis gaubae Bornm.

Sisymbrium irio L.

Thlaspi hastulatum (Stev.) ex DC.

Campanulaceae

Asyneuma amplexicaule Hand.-Mazz.

subsb. amplexicaule

Asyneuma pulchellum Bornm.

Campanula glomerata $\mathrm{L}$.

Campanula stevenii M.Bieb.

Caprifoliaceae

Lonicera floribunda Boiss. \& Buhse.

Caryophyllaceae

Acantholimon hohenackeriLedeb.

Arenaria alsinoides Willd.

Arenaria gypsophiloides L.

Arenaria insignis Litw.

Cerastium dichotomum $\mathrm{L}$.

Cerastium purpurascens Adams. var.

elbursense (Boiss.) Moschl.

Dianthus orientalis subsp. stenocalyx

Herniaria glabra L. var. glaberrima

Fenzl. in Ledeb.

Herniaria hirsuta L.

Herniaria incani Lam.

Minuartia lineata (Boiss.) Bornm.

Minuartia recurva Schinz. \& Thellung.

Minuartia sclerantha (Fisch. \& C.A.

Mey.) Thellung.

Petrohagia alpina (Habl.) Ball \& Heywood. Hem

Silene aucheriana Boiss.

Silene bupleuroides L.

Silene latifolia Poir.

Silene marschallii C.A. Mey. subsp.

sahendica (Boiss. \& Buhse) Malzh.

Stellaria alsinoides Boiss. \& Buhse.

$\begin{array}{ll}\text { Hem } & 2800-4000 \\ \text { Hem } & 2200-3700 \\ \text { Hem } & 3400-3600\end{array}$

$\begin{array}{ll}\mathrm{Ph} & 2200 \\ \mathrm{Hem} & 2400\end{array}$

Hem 3000-3300

Ch 2000-2300

Hem 2200

Hem 2000-3500

Hem 2000-3500

Hem 4300-4400

Hem 2000-3700

Hem 2000-2800

Thr 2000-2300

Thr 2700

Ch 2700-3500

Hem 2500-2600

Hem 2200

Hem 2000

2000-2900

2700

Hem

2100-2900

Hem

Thr

Thr

Hem

Thr

Hem

Thr

Hem

Hem

Hem

Thr

Thr

Hem

Hem

Hem

Hem

2500-4000

2700-4400

2700

2100-2600

4200-4500

2200-4200

2000-2200

2700-4100

2300-3700

2200-2400

2000-3400

2600-3500

2400-2600

3600

2600-3700

Ch

2500

$\mathrm{Ch}$

Thr

Hem

Hem

Thr

Hem

Hem

Hem

Thr

Hem

Hem

Hem

Thr

Hem

Hem

Hem

Hem

Thr

2600-3300

$3700-4300$

2200-2700

2000

2100-2600

3000-4000

2000-3300

2500-3800

3400-4300

2000-2200

2100

2500-3600

2900-3500

2100-3500

2800-3400

2400-2700
2000-2300

Stellaria media (L.) Vill.

Chenopodiaceae

Chenopodium foliosum Aschers.

Thr

2000-2600

Cistaceae

Helianthemum nummularium Mill.

Hem

Convolvulaceae

Convolvulus arvensis L. Hem

Crassulaceae

Rosularia sempervivum (M.Bieb.) A.Berger. Hem

Sedum lenkoranicum Grossh. Hem

Sedum pallidum M.B.

Thr

Sedum spurium M.Bieb.

Hem

Sempervivum iranicum Bornm. \& Gauba. Hem

Euphorbiaceae

Euphorbia szovitsii Fisch. \& Mey. Thr

Fabaceae

Astragalus (Sect. Adiaspastus) aureus $\quad$ Ch $\quad 2000-3000$

(Willd.)

Astragalus (Sect. Adiaspastus)

Ch $\quad 3400-4300$

macrosemius Boiss. \& Hohen.

Astragalus (Sect. Caprini) aegobromus Hem 2100-3700

Boiss. \& Hohen. in Boiss.

Astragalus (Sect. Caprini) chrysanthus Hem 2900-3300

Boiss. \& Hohen.

Astragalus (Sect. Hololeuce) alyssoides Lam. Hem

Astragalus (Sect. Hymenostegis) Ch

naftabensis Širj. \& Rech.f.

Astragalus (Sect. Hyppoglottidei)

Hem

atricapilus Bornm.

Astragalus (Sect. Hyppoglottidei) nurensis Hem

Boiss. \& Buhse.

Astragalus (Sect. Incani) askius Bunge. Hem

Astragalus (sect. Malacothrix) beckii Bornm. Hem

Astragalus (Sect. Ornithopodium) Hem

ornithopodioides Lam.

Astragalus (Sect. Theiochrus) siliqusus Hem

Astragalus microcephalus Willd. subsp. $\quad \mathrm{Ch}$

microcephalus

Coronilla orientalis Mill.

Medicago lupulina $\mathrm{L}$.

Medicago minima (L.) Bartalini.

Hem

Thr

Thr

Medicago rigidula (L.) All. Approx. var. Thr

submitis (Boiss.) Heyn.

Medicago sativa $\mathrm{L}$.

Onobrychis cornuta (L.) Desv.

Hem

$\mathrm{Ch}$

Oxytropis kotschyana Boiss. \& Hohen. Hem

Oxytropis persica Boiss.

Trifolium arvense $\mathrm{L}$. var. arvense

Trifolium canescens Willd.

Trifolium nigrescens Viv. subsp.

petrescens (Clem.) Holmboe.

Hem

Thr

Hem

Thr

3200-4100

2900-3300

$3700-4100$

2600

2500-3200

2400-3600

3100

2600

2200-2400

2000-2600

2000-2700

2000

2100

2200-2600

2600-3600

2800-3600

4000-4100

2000-2300

2000-2100

2000-2900
Trifolium repens $\mathrm{L}$.

Fumariaceae

Corydalis angustifoliaDC.

Hem

Corydalis verticillaris subsp. verticillaris Hem

Fumaria vaillantii Loiseh. in Desv. Thr

Gentianaceae

Gentiana septemfida Pall. Hem

2200

3300-3500

2200-2300

3700

Geraniaceae

Erodium cicutarium (L.) Lher. ex Aiton. Hem

Geranium lucidum L.

Thr

Hem

Hypericaceae

Hypericum armenum Jaub.

Hypericum scabrum $\mathrm{L}$.

Hem

Lamiaceae

Ajuga comata Stapf. Hem

Betonica nivea Stev. subsp. mazandarana $\mathrm{Ch}$

(Bornm.) Rech.f.
2800

2000-2200

2000-3000

2200-2900

3000

2000-2600

2600-2700

2500-2600

2900-3700 


\begin{tabular}{|c|c|c|}
\hline Dracocephalum aucheri Boiss. & Hem & $4300-4500$ \\
\hline Dracocephalum kotschyi Boiss. & Hem & 3000 \\
\hline Dracocephalum thymiflorum $\mathrm{L}$. & Thr & $2400-2700$ \\
\hline $\begin{array}{l}\text { Lallemantia iberica (Stev.) Fisch. \& C.A. } \\
\text { Mey. }\end{array}$ & Thr & 2700 \\
\hline Lamium albumL. subsp. album & Hem & $2800-3100$ \\
\hline Lamium amplexicaule $\mathrm{L}$. & Thr & $2000-2200$ \\
\hline Leonorus cardiaca L. subsp. cardiaca & $\mathrm{Ch}$ & 3700 \\
\hline Marrubium astracanicum Jacq. & $\mathrm{Ch}$ & $2100-3300$ \\
\hline Nepeta racemosa Lam. & $\mathrm{Ch}$ & $2000-3700$ \\
\hline Phlomis anisodonta Boiss. & $\mathrm{Ch}$ & $2000-3200$ \\
\hline Phlomis olivieri Benth. & $\mathrm{Ch}$ & $2300-2600$ \\
\hline Salvia atropatana Bunge. & Hem & $2000-3200$ \\
\hline Salvia sclarea $\mathrm{L}$. & Hem & 2500 \\
\hline Scutellaria glechomoides Boiss. & Hem & 4200 \\
\hline $\begin{array}{l}\text { Scutellaria pinnatifida A. Hamilt. subsp. } \\
\text { pinnatifida }\end{array}$ & Hem & $2000-3200$ \\
\hline $\begin{array}{l}\text { Stachys balansae Boiss. \& Kotschy ex } \\
\text { Boiss. }\end{array}$ & $\mathrm{Ch}$ & 2200 \\
\hline Stachys byzantina K.Koch. & $\mathrm{Ch}$ & $2000-2900$ \\
\hline Stachys lavandulifolia Vahl. & Hem & $2500-3500$ \\
\hline $\begin{array}{l}\text { Teucrium chamaedrys L. subsp. } \\
\text { syspirense (C.Koch.) Rech.f. }\end{array}$ & Hem & $2200-2300$ \\
\hline Teucrium polium $\mathrm{L}$. & Hem & $2000-2800$ \\
\hline Thymus fedtschenkoi Ronniger. & Hem & $2600-4100$ \\
\hline Thymus kotschyanus Boiss. \& Hohen. & Hem & $2200-3900$ \\
\hline $\begin{array}{l}\text { Ziziphora clinopoides Lam. subsp. } \\
\text { elbursensis (Rech.f) Rech.f. }\end{array}$ & Hem & $3000-4100$ \\
\hline \multicolumn{3}{|l|}{ Linaceae } \\
\hline $\begin{array}{l}\text { Linum nervosum Waldst. \& Kit. var. } \\
\text { bungei ( Boiss.) Sharifnia }\end{array}$ & Hem & $2500-3100$ \\
\hline \multicolumn{3}{|l|}{ Malvaceae } \\
\hline Alcea $\mathrm{sp}$. & $\mathrm{Ch}$ & 2000 \\
\hline Malva neglecta Wallr. & Hem & $2100-2700$ \\
\hline \multicolumn{3}{|l|}{ Orobanchaceae } \\
\hline Orobanche sp. & Hem & 2600 \\
\hline \multicolumn{3}{|l|}{ Papaveraceae } \\
\hline Papaver armeniacum (L.) DC. & Hem & $2300-2900$ \\
\hline Papaver fugax Poir. & Hem & $2200-3700$ \\
\hline \multicolumn{3}{|l|}{ Plantaginaceae } \\
\hline Linaria elymaitica (Boiss.) Kuprian. & Hem & $2100-3400$ \\
\hline Plantago atrata Hoppe. & Hem & $2000-4100$ \\
\hline Plantago lanceolata $\mathrm{L}$. & Hem & 2200 \\
\hline \multicolumn{3}{|l|}{ Polygonaceae } \\
\hline Oxyria digyna (L.) Hill & Hem & 4200 \\
\hline Polygonum rottboellioides Jaub. \& Spach. & Hem & $2400-3000$ \\
\hline Polygonum serpyllaceum Jaub. \& Spach. & Hem & $2400-4200$ \\
\hline Rumex chalepensis Miller. & $\mathrm{Ch}$ & $2000-2700$ \\
\hline Rumex elbursensis Boiss. & $\mathrm{Ch}$ & $2900-3600$ \\
\hline Rumex scutatus L. & Hem & $2100-2800$ \\
\hline \multicolumn{3}{|l|}{ Primulaceae } \\
\hline Androsace maxima $\mathrm{L}$. & Hem & $2000-3900$ \\
\hline Androsace villosa $\mathrm{L}$. & Hem & $2600-4300$ \\
\hline Primula macrocalyx Bunge. & Hem & $2000-2600$ \\
\hline \multicolumn{3}{|l|}{ Ranunculaceae } \\
\hline Ceratocephala testiculata (Crantz) Roth. & Thr & $2000-2300$ \\
\hline Delphinium aquilegifolium (Boiss.) Bornm. & $\mathrm{Ch}$ & 2100 \\
\hline Delphinium lanigerum Boiss. & $\mathrm{Ch}$ & $2100-3000$ \\
\hline Ficaria kochii (Ledeb.) Iransharh. \& Rech. & Hem & $2800-3500$ \\
\hline $\begin{array}{l}\text { Paraquilegia caespitosa J.R.Drumm. \& } \\
\text { Hutch. }\end{array}$ & Hem & 4200 \\
\hline Ranunculus crymophilus Boiss. \& Hohen. & Hem & $3900-4300$ \\
\hline Ranunculus repens $\mathrm{L}$. & Hem & $2900-3600$ \\
\hline Thalictrum foetidum $\mathrm{L}$. & Hem & $2400-2900$ \\
\hline \multicolumn{3}{|l|}{ Rosaceae } \\
\hline Cerasus microcarpa (C. A. Mey.) Boiss. & $\mathrm{Ph}$ & $2100-3000$ \\
\hline Crataegus microphylla K.Koch. & $\mathrm{Ph}$ & $2000-2200$ \\
\hline Crataegus sp. & $\mathrm{Ph}$ & $2000-2100$ \\
\hline Geum urbanum $\mathrm{L}$. & Hem & 2100 \\
\hline $\begin{array}{l}\text { Potentilla adscharica Sommier. \& Levier. } \\
\text { ex Keller. }\end{array}$ & Hem & 2900 \\
\hline
\end{tabular}

\begin{tabular}{llc}
\hline Potentilla argentea L. & Hem & 3000 \\
Potentilla bungei Boiss. & Hem & $2000-3100$ \\
Potentilla hirta L. & Hem & 2100 \\
Potentilla hololeuca Boiss. & Hem & 4300 \\
Potentilla iranica (Rech.) Schiman-Czeika Hem & $2100-3200$ \\
Potentilla polyschista Boiss. \& Hohen. & Hem & $4300-4400$ \\
Potentilla reptans L. & Hem & $2600-2900$ \\
Potentilla sp. & Hem & 3200 \\
Prunus divaricata Ledeb. & Ph & $2000-2500$ \\
Rosa iberica Stev. in Bieb. & Ch & $2000-2900$ \\
Sanguisorba minor Scop. subsp. muricata & Hem & $2000-2800$ \\
(Spach.) Briq. & & \\
Rubiace & &
\end{tabular}

(Spach.) Briq.

\section{Rubiaceae}

Asperua glomerata subsp. bracteata Hem 3800

Asperula setosa Jaub. \& Spach. Hem 2400-3200

Crucianella suaveolens C.A. Mey. $\quad$ Hem 2000-3200

Cruciata taurica (Pall. ex Willd.) Ehrend. Hem 2100-2200

Galium aparine L. $\quad$ Thr 2200-2700

Galium aucheri Boiss. Hem 4000-4400

$\begin{array}{lll}\text { Galium ghilanicum Stapf. } & \text { Thr } & 2000-2400\end{array}$

Galium hyrcanicum C.A.Mey. $\quad$ Hem 2100-3900

Galium spurim L. $\quad$ Thr 2000-3000

Galium verum L. subsp. glabrescens Ehrend. Hem 2000-2900

Saxifragaceae

Saxifraga exarata Vill. subsp. moschata Hem 2800-2900

$\begin{array}{lll}\text { Saxifraga iranica Bornm. } & \text { Hem } & 3200\end{array}$

Scrophulariaceae

Pedicularis caucasica M.Bieb. Hem 3400-4000

Pedicularis condensata M. Bieb. Hem 2600-3400

Pedicularis pycnantha Boiss. $\quad$ Hem $3500-3700$

Scrophularia amplexicaulis Benth. Hem 2900-3700

Scrophularia elbursensis Bornm. Hem 2100-2600

Scrophularia variegata M. Bieb. subsp. Hem 2100-3700

variegata

Verbascum speciosum Schrad. $\quad$ Ch 2000-3000

$\begin{array}{lll}\text { Veronica aucheri Boiss. } & \text { Thr } & 3900-4500\end{array}$

Veronica biloba Schreb. $\quad$ Thr 2100-3800

$\begin{array}{lll}\text { Veronica hederifolia L. } & \text { Thr } & 2000-2300\end{array}$

Veronica kurdica Benth. subsp. kurdica Hem 2500-4100

Veronica paederotae Boiss. $\quad$ Hem 4200-4300

Veronica persica Poir. Thr 2100-2600

Veronica rechingeri M.A.Fisch. Hem 2000-3600

Veronica verna $\mathrm{L}$. $\quad \mathrm{Thr} \quad 2000-2700$

Urticaceae

Urtica dioica $\mathrm{L}$. Ch $2200-2300$

Valerianaceae

Valeriana sisymbriifolia Vahl. $\quad$ Hem 2500-3700

Violaceae

Viola alba Besser. Hem 2100-3100

Viola occulta Lehm. $\quad$ Thr 2200-3200

Note: Ph: Phanerophyte, Ch: Chamaephyte, Ge: Geophyte: Hem: Hemicryptophyte, Thr: Therophytes

Table 2. Comparing the percentage of taxa within different plant groups along two altitudinal transects in Mt. Rostam-Nisht and Kheyrud forest

\begin{tabular}{|c|c|c|c|c|}
\hline Plant & Eudicots & Monocots & Gymnosperms & Pteridophytes \\
\hline
\end{tabular}

\section{Mt. Rostam-Nisht}

$\begin{array}{lcccc}\text { Families } & 75 & 14.58 & 2.08 & 8.33 \\ \text { Genera } & 82.74 & 13.69 & 0.6 & 2.98 \\ \text { Species } & 81.94 & 15.72 & 0.33 & 2.01\end{array}$

Kheyrud forest

$\begin{array}{lrrrr}\text { Families } & 72.15 & 13.92 & 1.27 & 12.66 \\ \text { Genera } & 73.53 & 18.63 & 0.49 & 7.35 \\ \text { Species } & 73.22 & 17.97 & 0.34 & 8.14\end{array}$




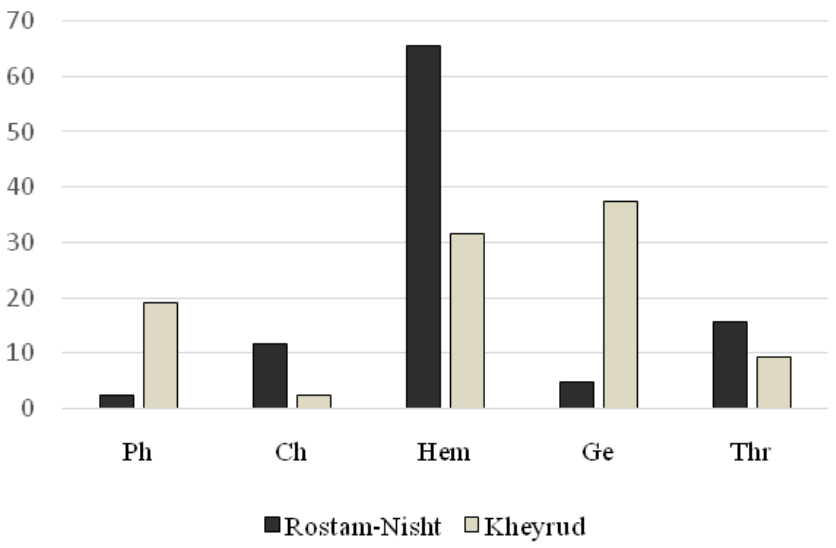

Figure 2. The comparison of life form spectrum in Mt. RostamNisht and Kheyrud forest

The similarities and differences of floristic comparison of the vegetation in Mt. Rostam-Nisht and Kheyrud forest were notable. Our findings represented close number of taxa, i.e. 299 taxa in the mountain and 295 taxa in the forest, but the number of different plant groups was different. As mentioned, pteridophytes were high in the forest comparing to the sub-alpine and alpine areas. Besides, the number of families and genera were less in the Mt. Rostam-Nisht while the species-rich genera were less in the forest (Table 2). The genera with high number of species occurred in Mt. Rostam-Nisht, e.g. the genus Astragalus with 13 taxa $(7.74 \%)$ represented the richest genus in our studied area, while the genus Carex with 8 taxa $(2.71 \%)$ was found as the richest genus in Kheyrud forest. Furthermore, there were 37 genera (12.54\%) with two taxa and 149 genera $(50.51 \%)$ with only one taxon in Kheyrud forest, while there were 37 genera (12.37\%) with two taxa and 100 genera $(33.44 \%)$ with only one taxon in the Mt. Rostam-Nisht.

The comparison of diversity in Mt. Rostam-Nisht and Kheyrud forest is manifest not only in the floristic composition, but also in terms of life forms of the plants. Life form reflects the adaptive responses of plants to environment and climate (Archibold 1995) and provides differences of various vegetation types (Neilson 1993; Archibold 1995). The dominant life forms were hemicryptophytes (196 taxa, 65.55\%) followed by therophytes (47 taxa, $15.72 \%$ ) and chamaephytes (35 taxa, $11.71 \%)$. Geophytes (14 taxa,4.68\%) and phanerophytes (7 taxa, $2.34 \%$ ) contained low proportion of life forms in the studied transect (Figure 2).

The comparison of life form spectrum between mountain and forest displayed an obvious difference in the proportion of phanerophytes. They were more in the forest than in Mt. Rostam-Nisht (19.2\% vs. $2.34 \%)$, while the occurrence of chamaephytes $(11.71 \%$ vs. $1.4 \%)$ and hemicryptophytes $(65.55 \%$ vs. $30.6 \%)$ was higher in our studied area than in Kheyrud forest. Interestingly, the geophytes contained only $4.68 \%$ of the flora in sub-alpine and alpine areas, whereas they confined higher proportion $(37.5 \%)$ in the forest. Finally, therophytes were more common in Mt. Rostam-Nisht (15.72\%) than in the forest
$(9.3 \%)$ (Figure 2). In this regard, phanerophytes in Kheyrud forest are a common group which decreases with altitude and are scattered at forest/steppe ecotones, and finally disappeared at altitudes higher than $2400 \mathrm{~m}$. Although, climatic stresses such as winter snow cover is supposed to filter out trees in high altitudes (Körner 1999) (see Moradi et al. 2017 for more details), but Noroozi and Körner (2018) believed that the absence of trees up to 2850 $\mathrm{m}$ is best explained by millennia of detrimental land use practices. Chamaephytes, a well-known life form in the mountains, with a long altitudinal range from 2800-4300 m in Mt. Rostam-Nisht, seems to cope effectively with grazing in lower altitudes and climatic harshness of high altitudes (Moradi et al. 2017).

The major variation of life form proportions across two transects is also related to geophytes and hemicryptophytes. Generally, geophytes comprise little number of species in alpine zones (Noroozi et al. 2011) as in our studied area. The sub-alpine and alpine zones are commonly characterized by hemicryptophytes (Noroozi et al. 2008) which are associated with colder climates and longer periods of coldness (Raunkiaer 1934). The activities such as human effects, grazing and disturbance can make suitable habitats for therophytes in both Mt. Rostam-Nisht and Kheyrud forest. Therophytes have been found as indicators for highly stressful habitat in the forests (Siadati et al. 2010; Naqinezhad et al. 2015). Similarly, Mt. Rostam-Nisht, particularly in lower altitudes is threatened by land use changes, strong grazing pressure and trampling which resulted to the growth of some annual species such as Alyssum minus, Descurainia sophia, Sisymbrium irio and Thlaspi hastulatum.

\section{Threat and conservation}

The changing of vegetation types and habitats from Hyrcanian forest to the timberline and the particular vegetation of the forest/steppe, makes the Alborz an important landscape for conservation of species and biodiversity. The importance is more when treeline ecotones between forest and steppe are discussed. Climatic warming might cause an upslope ascent of treeline ecotones and the consequent contraction and fragmentation of alpine areas (Camarero et al. 2006). So, treeline ecotones can be considered as early indicators of future changes and the stability of forest stands under the increasing stresses of climate change (Walker et al. 2003). Although, the ecotones and alpine areas of the Alborz are naturally more protected comparing to the forests, but they supply a large amount of food capacity for cattle, so is highly under the pressure of sheep grazing mainly in last decades. Human and agricultural activities, livestock grazing, severe harvesting of endangered species for economic and ornamental purposes as well as the collection of medical plants are the main threats in the Hyrcanian forest (Naqinezhad et al. 2017). On the other hand, overgrazing of the vegetation in the delicate sub-alpine and alpine areas caused destruction in vegetation, habitat and biodiversity loss and intensive reduction in space for endemic and narrow distributed species (Noroozi et al. 2008). The Alborz Mountains should receive considerable ecological 
attention for both conservation and theoretical reasons. More preserving and conservation of species and habitat is needed to protect its diverse and distinctive flora and vegetation.

\section{ACKNOWLEDGEMENTS}

This research was a part of Ph.D. dissertation which was supported by the University of Tehran, Tehran, Iran. The authors would like to express special thanks to A. Darvishsefat (Khazar Mountain Team, Chalus), and the Vandarbon mountaineering federation staffs for their assistance during field visits. Particular thanks to Prof. Dr. Sh. Zarre (University of Tehran) for determination and confirmation of several Astragalus species. The authors are also indebted to DAAD (Deutscher Akademischer Austausch Dienst) for a grant to H.M. (funding grant number 57214227).

\section{REFERENCES}

Akhani H, Mahdavi P, NorooziJ, Zarrinpour V. 2013. Vegetation patterns of the Irano-Turanian steppe along a $3000 \mathrm{~m}$ altitudinal gradient in the Alborz mountains of northern Iran. Folia Geobot 48: 229-255.

Archibold OW. 1995 Ecology of World Vegetation. Chapman \& Hall, London.

Assadi M, Massoumi AA, Khatamsaz M, Mozaffarian M. 1988-2015. Flora of Iran. Research Institute of Forests and Rangelands Publication, Teheran. [Persian]

Box EU. 1981. Macroclimate and plant forms: an introduction to predictive modeling in phytogeography. Dr. Junk W, The Hague.

Camarero JJ, Gutierrez E, Fortin MJ. 2006. Spatial patterns of plant richness across treeline ecotones in the Pyrenees reveal different locations for richness and tree cover boundaries. Glob Ecol Biogeogr 15: 182-191.

Frey W, Probst W. 1986. A synopsis of the vegetation of Iran. In: Kürschner H (ed.). Contribution of the Vegetation of Southwest Asia. Dr. Ludwig Reichert, Wiesbaden.

Gholizadeh H, Saeidi Mehrvarz SH, Naqinezhad A. 2017. Floristic study of the pure beech (Fagus orientalis Lipsky) stands in eastern Guilan, Iran. Nova Biologica Reperta 4 (3): 271-280.

Jafari SM, Akhani H. 2008. Plants of Jahan Nama protected area, Golestan province. N Iran. Pak J Bot 40: 1533-1554.

Jafari SM, ZarreSH, Alavipanah SK. 2013. Woody species diversity and forest structure from lowland to montane forest in Hyrcanian forest ecoregion. J Mt Sci 10: 609-620.

Khalili A. 1973. Precipitation patterns of central Elburz. Arch. Met. Geoph. Biokl Ser B. 21: 215-232.

Khan Sh, Page S, Ahmad H, Shaheen H, Harper D. 2012. Vegetation dynamics in the western Himalayas, diversity indices and climate change. Sci Tech Dev. 31 (3) : 232-243.

Klein JC. 1982. The chionophilic groups of the Central Alborz (Iran). Comparison with Central Asian counterparts. Phytocoenologia 10: 463-486.[French].

Klein JC.1987. The xerophilic highland lawns of the southern flanc of the central Alborz (Iran).Phytocoenologia 15: 253-280. [French]

Körner C. 1999. Alpine Plant Life: functional plant ecology of high mountain ecosystems. Springer, Heidelberg.

Körner C.2000. Why are there global gradients in species richness? Mountains might hold the answer. Trends Ecol Evol 15: 513-514.

Körner C. 2007. The use of "altitude" in ecological research. Trends Ecol Evol 22: 569-574.
Mahdavi P, Akhani H, Van der Maarel E. 2013. Species diversity and lifeform patterns in steppe vegetation along a $3000 \mathrm{~m}$ altitudinal gradient in the Alborz mountains, Iran.Folia Geobot 48: 7-22.

McCainCM. 2007. Could temperature and water availability drive elevational species richness patterns? A global case study for bats. Global Ecol Biogeogr 16: 1-13.

Mc Cain CM. 2009. Vertebrate range sizes indicate that mountains maybe higher in the tropics. Ecol Lett 12: 550-560.

McNeely JA, Miller KR, Reid WV, Mittermeier RA, Werner TB. 1990. Conserving the world biodiversity. IUCN, WRI, CI, WWF and World Bank, Washington D.C.

Moradi H, NaqinezhadA, Siadati S, Yousefi Y, Attar F, Etemad V, Reif A. 2016. Elevational gradient and vegetation environmental relationships in the central Hyrcanian forests of northern Iran.Nord J Bot 34: 1-14.

Moradi H, Attar F, Oldeland J. 2017. Plant functional type approach for a functional interpretation of altitudinal vegetation zones in the Alborz Mts., Iran.J. Mt. Sci 14: 2257-2269.

NaqinezhadA, Zare-Maivan H, Gholizadeh H. 2015. A floristic survey of the Hyrcanian forests in Northern Iran, using two lowland-mountain transects. J For Res 26: 187-199.

Naqinezhad A, Esmailpoor A. 2017. Flora and vegetation of rocky outcrops/cliffs near the Hyrcanian forest timberline, Mazandaran mountains, Northern Iran. Nord J Bot DOI: 10.1111/njb.01384.

Neilson RP. 1993. Transient ecotone response to climatic change; some and modelling approaches. Ecol Appl 3: 385-395.

Noroozi J, AkhaniH, Breckle SW. 2008. Biodiversity and phytogeography of the alpine flora of Iran. Biodiversity Conserv 17: 493-521.

Noroozi J, Pauli H, Grabherr G, Breckle SW. 2011.The subnival-nival vascular plant species of Iran: a unique high-mountain flora and its threat from Climate Warming. Biodivers Conserv 20:1319-1338.

Noroozi J. 2014. A glance at the Wildflowers of Iranian Mountains. Karimkhan Zand Publication, Tehran.

Noroozi J, Körner C. 2018. A bioclimatic characterization of high elevation habitats in the Alborz mountains of Iran. Alpine Bot. DOI: 10.1007/s00035-018-0202-9

Pant S, Samant SS. 2012. Diversity and regeneration status of tree species in Khokhan Wildlife Sanctuary, North-Western Himalaya. Trop Ecol 53: 317-331.

Parolly G. 2015. The High-Mountain Flora and Vegetation of the Western and Central Taurus Mts. (Turkey) in the Times of Climate Change. In: Ozturk M, Hakeem K, Faridah-Hanum I, Efe R (eds) Climate change impacts on high-altitude ecosystems. Springer, Cham.

Phillips OL, Martinez RV, Vargas PN, Monteagudo AL, Chuspe Zans ME, Sánchez WG, Cruz AP, TimanáM, Yli-Halla M, Rose S. 2003. Efficient plot-based floristic assessment of tropical forests. Trop Ecol 19: 629-645.

Qiong L, Grytnes JA, BBirks HJ. 2010. Alpine vegetation and speciesrichness patterns along two altitudinal gradients in the Gyama Valley, south-central Tibet, China. Plant Ecol Divers 3: 235-247.

Raunkiaer C. 1934. The Life Form of Plants and Statistical Plant Geography. Clarendon Press, Oxford.

Rechinger KH. 1963-2010. Flora Iranica, Vols. 1-176. Akademische Druck- und Verlagsanstalt, Graz.

Siadati S, Moradi H, Attar F, EtemadV, Hamzeh'ee B, Naqinezhad A. 2010. Botanical diversity of Hyrcanian forests; a case study of a transect in the Kheyrud protected lowland mountain forests in northern Iran. Phytotaxa 7: 1-18.

Shetekauri Sh, Chelidze D, Barnaveli N. 2012. Diversity and florogenesis of subnival flora of the Caucasus. J Life Sci. 6:917-930. Townsend CC, Guest E, Al-Rawi A. 1966-1985. Flora of Iraq, Vols. 1-10, Ministry of Agriculture of the Republic of Iraq, Baghdad.

Van der Maarel E. 2005. Vegetation Ecology. Blackwell Publishing, Oxford.

Walker S, Wilson JB, Steel J, Rapson GL, SmithB, King WM, Cottam YH. 2003. Properties of ecotones: Evidence from five ecotones objectively determined from a coastal vegetation gradient. J Veg Sci14: 579-590.

Zohary M. 1973. Geobotanical foundations of the Middle East, Vols. 1-2. Fischer Verlag, Stuttgart. 